\title{
Wide-angle stop-gap chalcogenide photonic crystals generated by direct multiple-line laser writing
}

\author{
E. Nicoletti · D. Bulla • B. Luther-Davies • M. Gu
}

Received: 21 June 2011 / Revised version: 17 August 2011 / Published online: 12 October 2011

(C) Springer-Verlag 2011

\begin{abstract}
We present the fabrication and the angle-resolved optical characterizations of three-dimensional chalcogenide photonic crystals with a wide-angle stop gap. Multiple-line scanning provides an effective remedy to the elongation of the focal spot in the $z$ direction during direct laser writing fabrication in high refractive index and highly nonlinear chalcogenide glasses. The aspect ratio of the rods is reduced from 4.46 to 1.53 , thus allowing the successful fabrication of three-dimensional chalcogenide photonic crystals with a face-centered cubic symmetry and quasi-circular rods. Suppression of the angle-resolved transmission spectra is observed at a wide range of incident angles.
\end{abstract}

\section{Introduction}

The direct laser writing (DLW) fabrication method has recently attracted a lot of interest in the photonics community. DLW is a technique that allows for the fabrication of small features in a photosensitive material, without the use of complex optical systems or photo-masks. In particular, writing based on two-photon absorption (TPA) has emerged as an attractive and versatile tool for generating three-dimensional (3D) microstructures [1-5]. DLW by two-photon polymerization (TTP) is a highly flexible technique that offers the

\section{E. Nicoletti · M. Gu (凶)}

Centre for Micro-Photonics and CUDOS, Faculty of Engineering and Industrial Sciences, Swinburne University of Technology, Hawthorn, VIC 3122, Australia

e-mail:mgu@swin.edu.au

D. Bulla $\cdot$ B. Luther-Davies

Laser Physics Centre \& CUDOS, Research School of Physical

Sciences and Engineering, Australian National University,

Canberra, ACT 0200, Australia unparalleled ability to create arbitrary photonic structures [6] for applications including microfluidics [7, 8], microoptics, micro-opto-electromechanical systems (MOEMS) [9] and biomedical devices [10]. The emergence of TPP has facilitated the fabrication of 3D photonic crystals (PhCs), which allows for the generation of 3D photonic bandgap (PBG) structures and thus 3D confinement and effective control of the flow of light.

Two types of photosensitive material have been adopted for 3D fabrication by TPA: polymer resins and chalcogenide glasses (ChGs). Low refractive-index polymer resins have shown their capabilities for some photonic applications [1, $3-5,11-13]$. However, the difference in refractive index between polymer and air is not great enough to open up wideangle 3D PBG, which is crucial for the practical use of the PhCs. Furthermore, the nonlinearity of polymers is not high enough to achieve nonlinear switching. To address these limitations, ChGs have been recently used for DLW via TPA [14-18]. ChGs have high refractive indices, which can potentially provide a complete bandgap for $\mathrm{PhCs}$. They exhibit high nonlinearities in the NIR wavelength region with Kerr nonlinear coefficients $\left(\chi^{3}\right)$ two to three orders of magnitude higher than those of silicate glasses [19-21]. They are highly transparent in the mid to far-infrared (IR) and have low characteristic phonon energies. In particular arsenic trisulphide $\left(\mathrm{As}_{2} \mathrm{~S}_{3}\right)$ films have been recently utilized as negative photoresist for the direct patterning of nonlinear microstructures $[15,16,18]$. The refractive index of the as deposited chalcogenide film is around 2.3-2.4 and it increase of 5.5\% after photo-polymerization [22].

Although two-photon-assisted DLW has been successfully used to fabricate $3 \mathrm{D}$ PhCs in $\mathrm{As}_{2} \mathrm{~S}_{3}[15,16,18]$, the utilization of a high numerical aperture (NA) objective has resulted in the inherent problem of the elongation of the focal spot along the axial direction. This is a hurdle toward the 


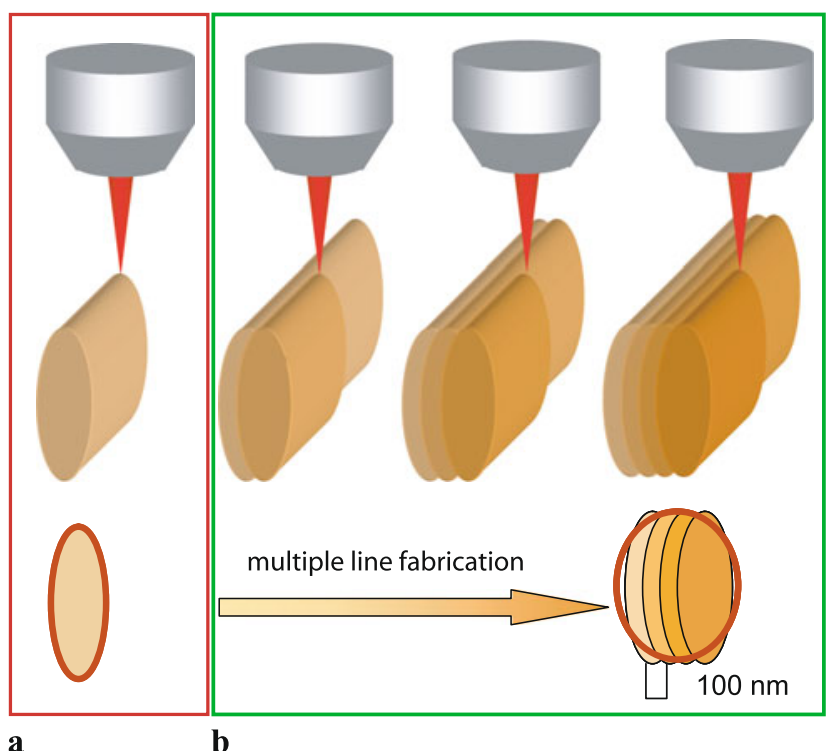

Fig. 1 (a) Sketch of the ellipsoidal shape of the rods due to the high NA objective used in the fabrication set up. (b) Schematic of the multiple-line fabrication method which provides a solution for reducing the elongation of the focal spot. Each rod is composed by four lines scanned with an offset between each other of $100 \mathrm{~nm}$

realization of 3D PhCs with a wide-angle bandgap. Though a multiple-rod scanning method was proposed to address the elongation issue [16], the reported experimental results failed to demonstrate the reduction of the aspect ratio of smooth rods in the construction of 3D PhCs and the existence of a wide-angle stop gap [16]. We here demonstrate the existence of a wide-angle stop gap in a woodpile $\mathrm{ChG} \mathrm{PhC}$ using the angle-resolved measurements. The problem of the focal spot elongation induced by the high NA objective can be solved by a direct multiple-line laser writing technique that allows for a reduction in the aspect ratio of the focal spot and hence permits the observation of a wide-angle stop gap.

\section{Direct multiple-line laser writing}

One of the diffraction features in the focal region of a high NA objective is the elongation of the focal spot [23]. As a result, the polymerized rods will have an ellipsoidal shape that prevents the achievement of a complete PBG (see Fig. 1(a)). In order to reduce the aspect ratio of the focal spot, we fabricated multiple lines side by side with an offset of $100 \mathrm{~nm}$, as illustrated in the inset in Fig. 1(b). This choice of offset allows for the fabrication of smooth rods with a reduced aspect ratio. The aspect ratio of the focal spot can be in this way easily reduced from 4.46 to 1.53 for a NA $=1.4$.

The photonic band structure was calculated using an iterative eigensolver program (RSoft, Bandsolve). The simulation engine employs the plane-wave expansion (PWE) algorithm to perform band computations. It is predicted, through

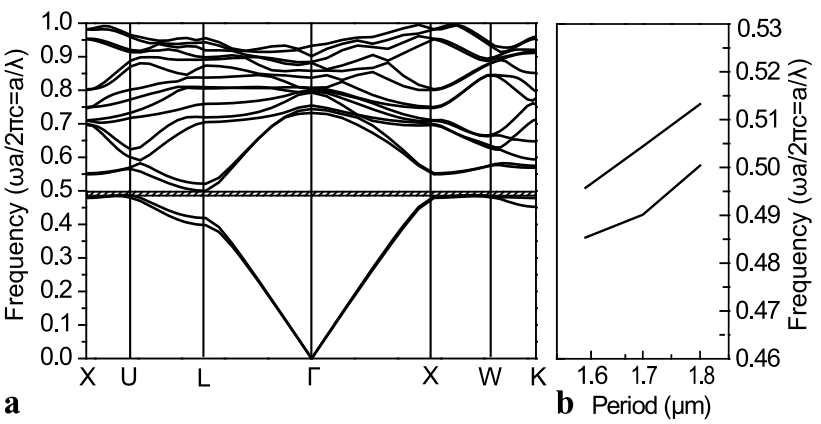

Fig. 2 (a) Calculated band structure of a 3D woodpile $\mathrm{PhC}$ with a FCC geometry and lattice parameters: in-plane spacing $(d x)=1.7 \mu \mathrm{m}$ and interlayer spacing $(d z)=0.6 \mu \mathrm{m}$. The thickness and aspect ratio of the rods used in the calculations are an estimation based on the multipleline fabrication conditions. (b) R-soft simulations

theoretical calculations, that a woodpile $\mathrm{PhC}$ with more circular rods arranged in a face-centred cubic (FCC)-like geometry, can lead to a complete PBG at a normalized frequency of about 0.5 (Fig. 2(a)). Further simulation confirms that, when the periodicity varies between $1.6 \mu \mathrm{m}$ and $1.8 \mu \mathrm{m}$, a complete PBG exists, as shown in Fig. 2(b).

\section{Experimental results}

A 3D PhC was fabricated in an $\mathrm{As}_{2} \mathrm{~S}_{3}$ film of $\sim 16-20 \mu \mathrm{m}$ in thickness, deposited onto glass slide substrates using thermal evaporation. To induce structural changes in the $\mathrm{ChG}$ film, we focused femtosecond laser pulses (with a pulse width of $\sim 100 \mathrm{fs}$ and a repetition rate of $1 \mathrm{kHz}$ ) operating at wavelength $800 \mathrm{~nm}$ into the glass with an oil immersion objective (Olympus, NA 1.4, 100×) [15]. The polymerized $\mathrm{PhCs}$ was developed with a multi-step etching process using a solution of diisopentylamine (Alfa Aesar) and DMSO (Sigma-Aldrich). The sample was first immersed in a concentrated solution ( $2 \mathrm{~mol} \%$ ) for about 3-4 minutes. A further immersion in a lower concentration solution $(0.1 \mathrm{~mol} \%)$ allows for the removal of the residual unpolymerized glass inside the 3D structures.

Figures 3(a) and (b) show the SEM images of an $\mathrm{As}_{2} \mathrm{~S}_{3}$ woodpile structure fabricated using our multiple scanning technique to produce a structure in accordance with the calculations. In the side-view SEM image (Fig. 3(c)) smooth rods with an almost circular cross-section can be observed, as expected. The $\mathrm{PhC}$ has a lattice constant $(d x)=1.7 \mu \mathrm{m}$ and interlayer spacing $(d z)=(\sqrt{2} / 4) \cdot a=0.6 \mu \mathrm{m}$ and it was fabricated with a laser beam power of $2 \mu \mathrm{W}$ and a scanning speed of $100 \mu \mathrm{m} / \mathrm{s}$.

Figures 3(d) and (e) show the SEM images of a woodpile structure obtained with the standard one-line scanning method. The comparison between the PhCs fabricated with the single and the four-line scanning approach demonstrates 

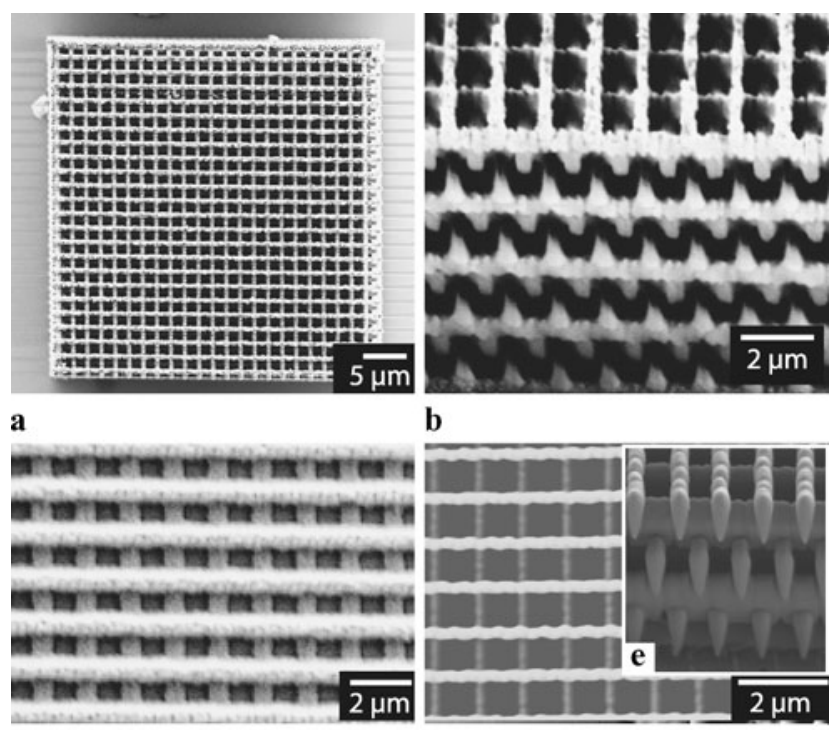

c

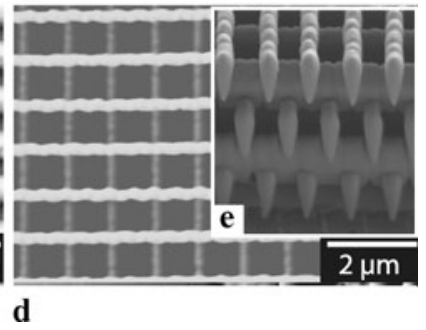

Fig. 3 (a, $\mathbf{b}$ and $\mathbf{c}) \mathrm{SEM}$ images of an $\mathrm{As}_{2} \mathrm{~S}_{3}$ woodpile structure, with lattice parameters $d x=1.7 \mu \mathrm{m}$ and $d z=0.6 \mu \mathrm{m}$, fabricated according to the calculation in Fig. 2; (a and c) top view (b) side view at an angle of $30^{\circ}$. (d and e) SEM images of an $\mathrm{As}_{2} \mathrm{~S}_{3}$ woodpile structure with lattice parameters $d x=1 \mu \mathrm{m}$ and $d z=0.6 \mu \mathrm{m}$, fabricated with a single-line direct laser writing method. (d) Top view, (c) side view at an angle of $35^{\circ}$

the improvements obtained with the multiple-line laser writing. Scanning four lines with an offset between each other of $100 \mathrm{~nm}$ allows for the fabrication of more homogeneous and circular rods with improved surface quality compared to the standard one-line scanning approach.

To confirm the theoretical calculations, we measured the FTIR transmission spectra of the fabricated $\mathrm{PhC}$. The optical characteristics of the 3D PhC were measured by using the Nicolet Nexus Fourier transform infrared (FTIR) spectrometer with a Continuum IR microscope (Thermo Nicolet, Madison, WI, USA). The FTIR spectrometer was used in two different configurations, as shown in Fig. 4. The reflective $32 \times$ NA 0.65 objective (Re echromat, Thermo Nicolet) provides a hollow light cone varying from $\theta_{\text {in }}=18^{\circ}$ to $\theta_{\text {out }}=41^{\circ}$. To achieve normal incidence and a smaller light cone $\left(\theta_{\text {mask }}\right)$ of $5^{\circ}$, the sample was tilted and a pinhole placed in front of the objective (Fig. 4(a)) to achieve angleresolved measurements. In this way the measurements can be accurately confined to a certain direction. When adopted the standard configuration, the sample was placed in a horizontal position (Fig. 4(b)) and the light was incident at a larger and broader angle [24].

Figure 5(a) shows the FTIR spectra for the 3D PhC in Fig. 3. The pinhole was first placed at normal incidence and the mask was then moved in the two opposite directions. Taking into account $\theta_{\text {mask }}$, we could measure the stop gap for angles varying from $+10.5^{\circ}$ to $-12.5^{\circ}$. For all the different angles it is possible to observe partial suppression in the

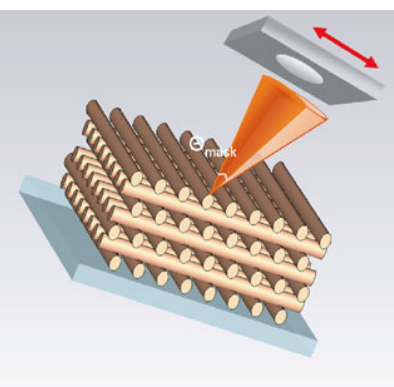

a

Fig. 4 (a) Modified FTIR measurement setup. The sample is tilted and a mask with a pinhole is placed in front of the objective. The configuration results in a reduced light cone $\left(\theta_{\text {mask }}\right)$ that allows for the normal incident direction and selective angles measurements. (b) Standard setups of the NA 0.65 reflective objective for FTIR characterizations of 3D PhCs. The objective is circularly symmetric around its axis with a hollow light cone $\theta_{\text {out }}-\theta_{\text {in }}$

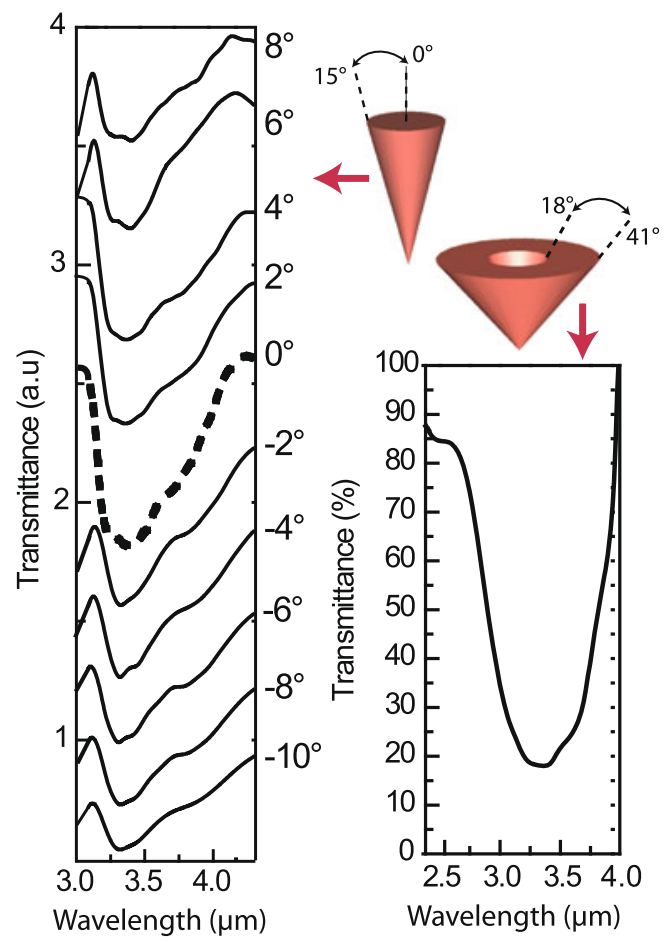

Fig. 5 FTIR spectra of the multiple-line woodpile PhC in Fig. 3 performed (a) at different angles, using pinhole-assisted objective setup and (b) using the standard configuration for the reflective objective

transmission at a wavelength of about $3.4 \mu \mathrm{m}$, in agreement with the band structure calculations. To account for larger angles, FTIR measurements in the standard reflective objective configuration have been performed. The FTIR spectrum in Fig. 5(b) shows a strong stop gap with a suppression in transmission of $82 \%$.

The FTIR spectra in Figs. 5(a) and (b) confirm our theoretical predictions of a $\mathrm{PBG}$ over a wide-angle range in the ChGs 3D PhCs. To the best of our knowledge, this is the first 
time that a wide-angle stop gap in high refractive-index and highly nonlinear PhCs could be observed. The observation of a wide-angle stop gap proves the successful fabrication of high quality $\mathrm{PhCs}$ with more circularly shaped rods.

\section{Conclusion}

In summary we have demonstrated wide-angle stop gaps in a 3D ChG PhC fabricated by a multiple-line scanning method to reduce the aspect ratio of the axially elongated rods. The band structure simulation for the 3D woodpile PhC stacked by the quasi-circular rods shows the existence of a complete PBG. The angle-resolved Fourier transform infrared (FTIR) measurements have confirmed the successful fabrication of PhCs that suppress the transmission in a wide range of incident angles, as predicted by the theory. These results are an important step towards the realization of multifunctional all-optical devices.

Acknowledgements This research was conducted by the Australian Research Council Centre of Excellence for Ultrahigh bandwidth Devices for Optical Systems (project number CE110001018).

\section{References}

1. M. Straub, M. Gu, Opt. Lett. 27, 1824 (2002)

2. M. Deubel, G. von Freymann, M. Wegener, S. Pereira, K. Busch, C.M. Soukoulis, Nat. Mater. 3, 444 (2004)

3. J. Serbin, A. Ovsianikov, B. Chichkov, Opt. Express 12, 5221 (2004)

4. J. Serbin, A. Egbert, A. Ostendorf, B.N. Chichkov, R. Houbertz, G. Domann, J. Schulz, C. Cronauer, L. Fröhlich, M. Popall, Opt. Lett. 28, 301 (2003)

5. H.B. Sun, S. Matsuo, H. Misawa, Appl. Phys. Lett. 74, 786 (1999)
6. H.-B. Sun, S. Kawata, in NMR. 3D Analysis. Photopolymerization (Springer, Berlin, 2004), pp. 169-273

7. Y. Liao, Y. Ju, L. Zhang, F. He, Q. Zhang, Y. Shen, D. Chen, Y. Cheng, Z. Xu, K. Sugioka, K. Midorikawa, Opt. Lett. 35, 3225 (2010)

8. H. Sun, F. He, Z. Zhou, Y. Cheng, Z. Xu, K. Sugioka, K. Midorikawa, Opt. Lett. 32, 1536 (2007)

9. L. Erdmann, A. Deparnay, G. Maschke, M. Längle, R. Brunner, J. Microlithogr. Microfabr., Microsyst. 4 (2005)

10. V.V. Kancharla, S. Chen, Biomed. Microdevices 4, 105 (2002)

11. B.H. Cumpston, S.P. Ananthavel, S. Barlow, D.L. Dyer, J.E. Ehrlich, L.L. Erskine, A.A. Heikal, S.M. Kuebler, I.-Y.S. Lee, D. McCord-Maughon, J. Qin, H. Röckel, M. Rumi, X.-L. Wu, S.R. Marder, J.W. Perry, Nature 398, 51 (1999)

12. S. Wu, J. Serbin, M. Gu, J. Photochem. Photobiol. A, Chem. 181, 1 (2006)

13. H.B. Sun, T. Kawakami, Y. Xu, J.Y. Ye, S. Matuso, H. Misawa, M. Miwa, R. Kaneko, Opt. Lett. 25, 1110 (2000)

14. A. Feigel, M. Veinger, B. Sfez, A. Arsh, M. Klebanov, V. Lyubin, Appl. Phys. Lett. 83, 4480 (2003)

15. E. Nicoletti, G. Zhou, B. Jia, M.J. Ventura, D. Bulla, B. LutherDavies, M. Gu, Opt. Lett. 33, 2311 (2008)

16. S. Wong, M. Deubel, F. Pérez-Willard, S. John, G.A. Ozin, M. von Wegener, Adv. Mater. 18, 265 (2006)

17. A. Feigel, Z. Kotler, B. Sfez, A. Arsh, M. Klebanov, V. Lyubin, Appl. Phys. Lett. 77, 3221 (2000)

18. E. Nicoletti, D. Bulla, B. Luther-Davies, M. Gu, Opt. Lett. 36, 2248 (2011)

19. D. Freeman, C. Grillet, M.W. Lee, C.L.C. Smith, Y. Ruan, A. Rode, M. Krolikowska, S. Tomljenovic-Hanic, C. de Sterke, M.J. Steel, B. Luther-Davies, S. Madden, D.J. Moss, Y.H. Lee, B.J. Eggleton, Photonics Nanostruct. Fundam. Appl. 6, 3 (2008)

20. D. Freeman, S. Madden, B. Luther-Davies, Opt. Express 13, 3079 (2005)

21. K. Paivasaari, V.K. Tikhomirov, J. Turunen, Opt. Express 15, 2336 (2007)

22. S.H.E. Wong, Arsenic Trisulfide Inorganic Photoresist for ThreeDimensional Photolithography (2008)

23. M. Gu, Advanced Optical Imaging Theory. Springer Series in Optical Sciences, vol. 75 (Springer, Berlin, 2000)

24. M.J. Ventura, M. Straub, M. Gu, Opt. Express 13, 2767 (2005) 